\title{
Identification of zebrafish magnetoreceptor and cryptochrome homologs
}

\author{
Zuoqiong Zhou ${ }^{1,2}$, Xiyang Peng ${ }^{1,2}$, Jianbin $\mathrm{Chen}^{2}$, Xiushan $\mathrm{Wu}^{1}$, Yuequn Wang ${ }^{*}$ \\ \& Yunhan Hong ${ }^{2 * *}$ \\ ${ }^{1}$ Ministry of Education Key Laboratory for Developmental Biology and Protein Chemistry, College of Life Sciences, Hunan Normal University, \\ Changsha 410081, China; \\ ${ }^{2}$ Department of Biological Sciences, National University of Singapore, Singapore 117543, Singapore
}

Received July 2, 2016; accepted August 3, 2016; published online September 06, 2016

\begin{abstract}
Magnetoreception is essential for magnetic orientation in animal migration. The molecular basis for magnetoreception has recently been elucidated in fruitfly as complexes between the magnetic receptor magnetoreceptor (MagR) and its ligand cryptochrome (Cry). MagR and Cry are present in the animal kingdom. However, it is unknown whether they perform a conserved role in diverse animals. Here we report the identification and expression of zebrafish MagR and Cry homologs towards understanding their roles in lower vertebrates. A single magr gene and 7 cry genes are present in the zebrafish genome. Zebrafish has four $c r y l$ genes (crylaa, crylab, crylba and crylbb) homologous to human CRY1 and a single ortholog of human CRY2 as well as 2 cry-like genes (cry4 and cry5). By RT-PCR, magr exhibited a high level of ubiquitous RNA expression in embryos and adult organs, whereas cry genes displayed differential embryonic and adult expression. Importantly, magr depletion did not produce apparent abnormalities in organogenesis. Taken together, magr and cry 2 exist as a single copy gene, whereas cry 1 exists as multiple gene duplicates in zebrafish. Our result suggests that magr may play a dispensable role in organogenesis and predicts a possibility to generate magr mutants for analyzing its role in zebrafish.
\end{abstract}

magnetoreception, MagR, cryptochrome, ortholog

Citation: Zhou, Z., Peng, X., Chen, J., Wu, X., Wang, Y., and Hong, Y. (2016). Identification of zebrafish magnetoreceptor and cryptochrome homologs. Sci China Life Sci 59, 1324-1331. doi: 10.1007/s11427-016-0195-x

\section{INTRODUCTION}

Migration or navigation over long distances is a common behavior in diverse animals (Bauer and Klaassen, 2013). Migration by the chum salmon for mating and spawning, for instance, covers up to $4,000 \mathrm{~km}$ from the British Columbia's coast to the river Grays freshwater area (Yano et al., 1997). The Arctic Tern annually migrates an estimated distance of over $40,000 \mathrm{~km}$ between the northern and southern hemispheres for breeding and wintering (Egevang et al., 2010; Newton, 2010). Mechanisms underlying orientation

*Corresponding author (email: yuequnwang@ @unnu.edu.cn)

**Corresponding author (email: dbshyh@nus.edu.sg) and migration have attracted considerable interest. Although it has been proposed that orientation and/or migration is dependent on many factors such as the Sun, the Moon (Dacke et al., 2003), polarised light (Cronin et al., 2006) and olfaction, an essential role of the Earth's magnetic field in orientation/migration has received increasing attention and wider acceptation (Kimchi et al., 2004). Extensive behavioral experiments show that animals have the ability to sense the earth's magnetic field for orientation and migration (Wiltschko and Wiltschko, 2006). This ability is called magnetoreception (MR) and is present in diverse animals ranging from invertebrates to vertebrates. Indeed, it has been documented that MR is necessary for orientation in diverse organisms such as the nematode (Vidal-Gadea et al., 
2015), fruitfly (Gegear et al., 2008), pigeon (Mora et al., 2004), bat (Holland et al., 2006) and woodmice (Mather and Baker, 1981). Therefore, MR appears to represent a conserved sense underlying orientation and migration across animal phyla.

The molecular basis of MR has recently been established in Drosophila (Qin et al., 2016). In this organism, a flavoprotein called cryptochrome (Cry) and a compass protein called magnetoreceptor (MagR) form complexes essential for perceiving geomagnetic information, whereas Crydeficient Drosophila does not show magnetosensitive behaviors (Gegear et al., 2008). The Cry-MagR complexes are capable of activation by magnets and sensing the surrounding magnetic field. Besides Drosophila, Cry is also considered to be a potential magnetoreceptor in many other species (Heyers et al., 2007; Liedvogel and Mouritsen, 2010).

Besides its essential role in animal orientation and migration, MR is also involved in certain physiological processes (Lai, 1996; Walleczek and Liburdy, 1990). In human, magnetotherapy is widely used to treat a variety of diseases including pain, musculoskeletal trauma, vascular and endocrine disorders, and has even led to the concept and practice of magnetomedicine (Markov, 2015; Wang and Wu, 1997). Understanding of mechanisms underlying magnet biology and physiology will provide invaluable information for magnetomedicine.

Laboratory fishes such as zebrafish (Danio rerio) and medaka are excellent vertebrate models for developmental biology and functional genomics. This study aimed at the identification of zebrafish magr and cry homologs as a prelude for magnet biology in this organism. We show that zebrafish has a single magr gene and 5 cry genes as well as
2 cry-like genes. All the zebrafish magr and cry genes exhibited ubiquitous RNA expression throughout life. Moreover, magr depletion through morpholino-mediated gene knockdown did not show strong impact on organogenesis and survival, suggesting the possibility of generating magr knockout zebrafish.

\section{RESULTS}

\section{Identification of zebrafish magr and cry genes}

In the zebrafish genome, there is a single magr gene predicting a protein of 129 amino acid residues. Multiple sequence alignment revealed that the predicted zebrafish MagR is maximally identical to MagR from other organisms including human (84\%) and Drosophila (74\%; Figure $\mathrm{S} 1$ in Supporting Information). A phylogenetic analysis clustered this protein with known MagR proteins (Figure 1A). Moreover, the zebrafish magr possesses a conserved genomic organization of human MAGR gene in that all exons of the coding sequence (CDS) share the same lengths (Figure 2A). Most importantly, a clear syntenic relationship exists between zebrafish and human in the magr-bearing chromosomal region (Figure 3). Thus, zebrafish magr is orthologous to the human MAGR.

A total of 7 cry genes were identified in the zebrafish genome, namely, crylaa, crylab, crylba, crylbb, cry2, cry 4 and cry5. Protein sequence alignment revealed that the protein products predicted by crylaa, crylab, crylba and $c r y 1 b b$ are maximally identical to Cry1 of other organisms including human (over 75\%) and Drosophila (over 37\%; Figure S2 in Supporting Information); zebrafish Cry2 is maximally identical to Cry2 of other organisms including
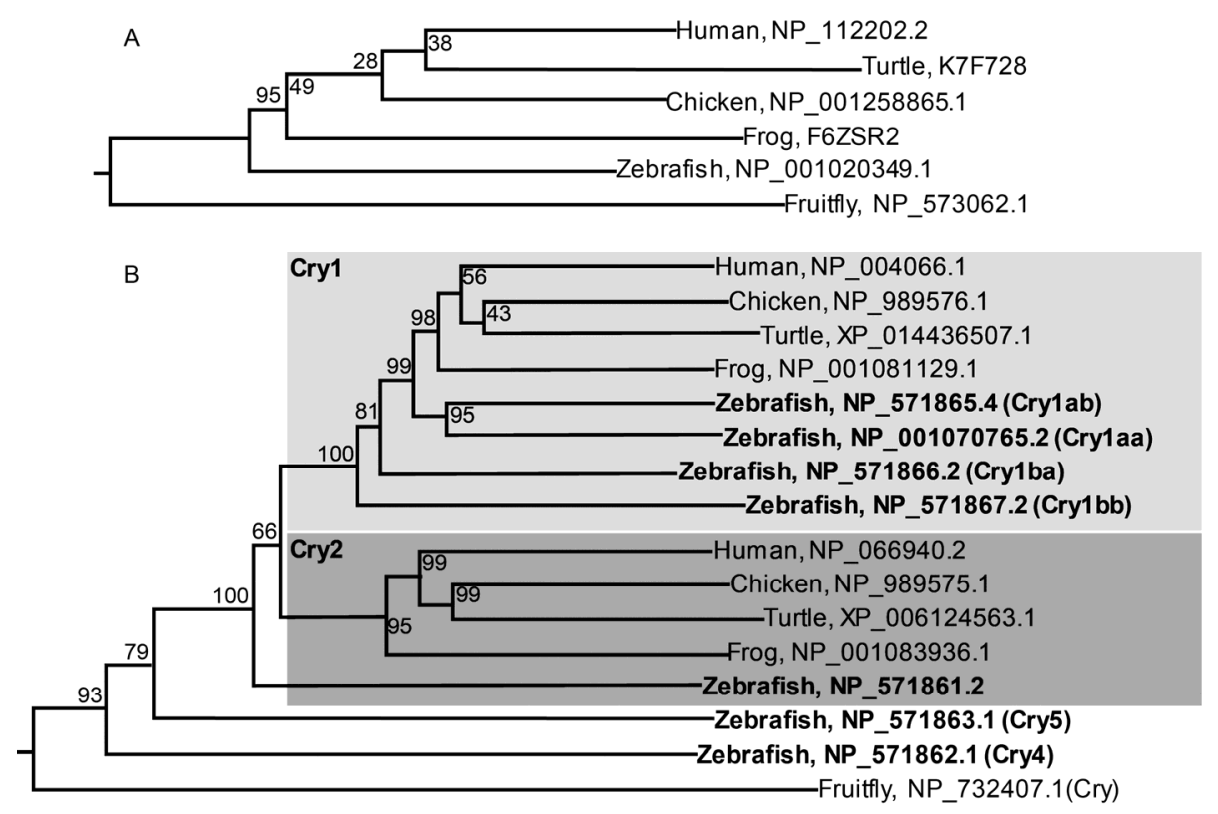

Figure 1 Phylogenetic tree of MagR and Cry proteins. The tree was generated by Neighbor-Joining algorithm. A, MagR proteins. B, Cry proteins. Sequence accession numbers are given following organisms. All zebrafish Cry proteins are remarkably in bold. Cry 1 and Cry 2 proteins are grouped in color. 


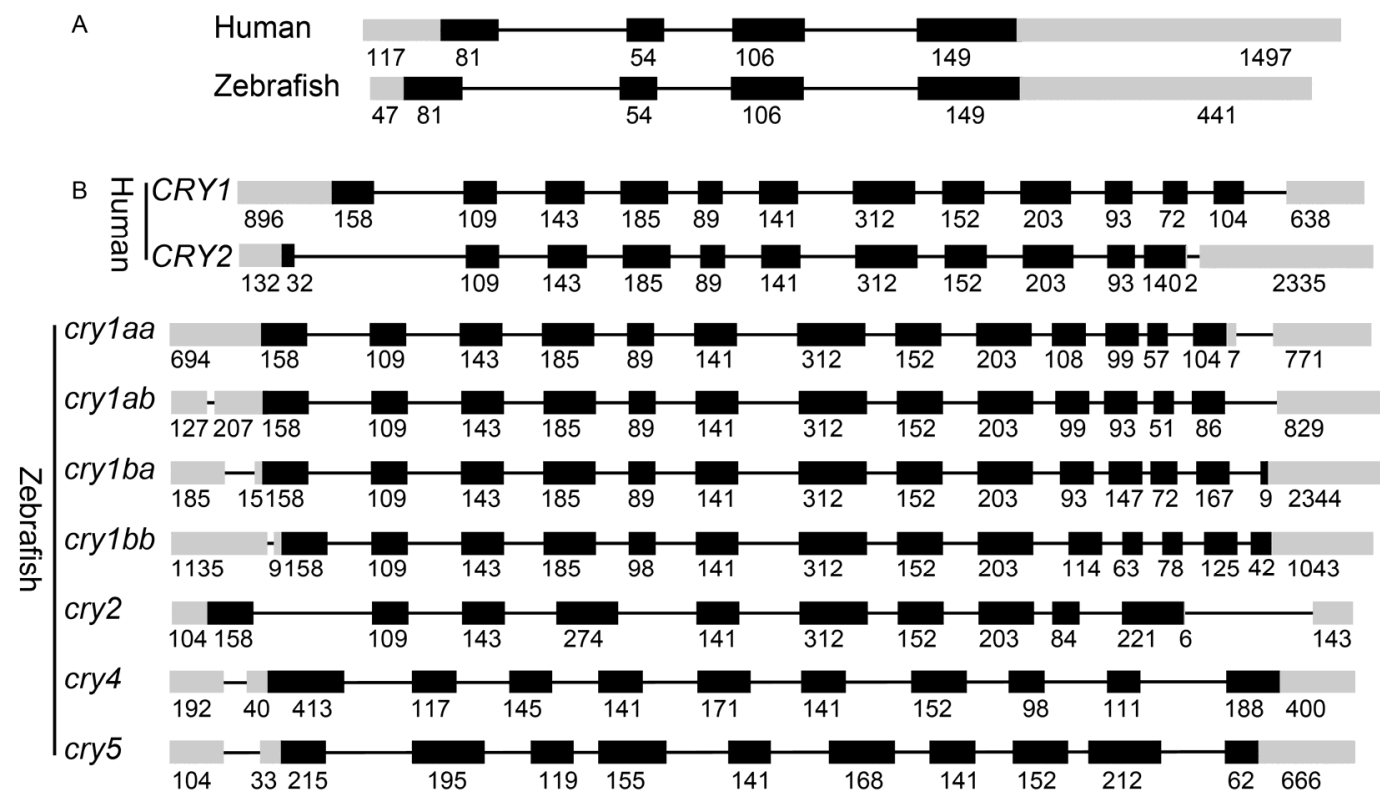

Figure 2 Gene structures of magr and cry genes in human and zebrafish. A, magr gene. B, cry genes. Exons are numbered with their sizes being given in bp. Notably, all cry genes, except for zebrafish cry4 and cry5, have highly conserved exon-intron structures. Grey box, UTR (untranslated region); black box, CDS; line, intron.

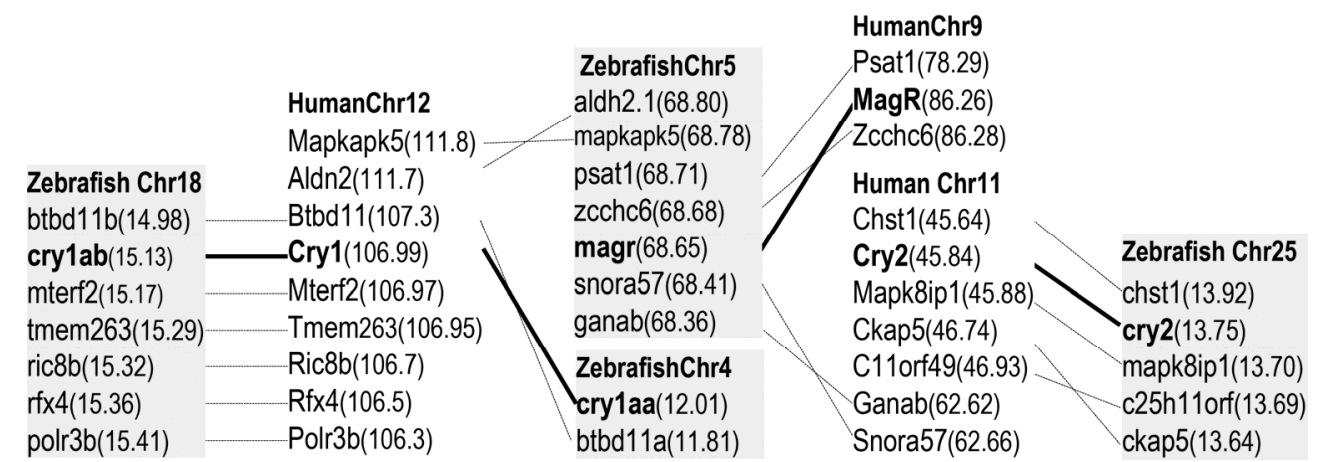

Figure 3 Chromosomal synteny of magr and cry genes. Shown are gene-bearing chromosomal regions that clearly have syntenic relationships between zebrafish and human. Parenthesis, chromosomal positions in million base pairs.

human $(79 \%)$ and Drosophila (40\%; Figure S2 in Supporting Information). The phylogenetic analysis displayed that Cry1 or Cry2 of different organisms were clustered together (Figure 1B). We also conducted splice site analysis of cry genes in zebrafish and human (Figure 2B). Notably, conserved exon structures were found in human $C R Y 1$ and all zebrafish cryl genes (crylaa, crylab, crylba and crylbb) with the first 9 exons of CDS sharing the same lengths, except for exon 5 in cry $1 b b$. Similarly, human $C R Y 2$ and zebrafish cry2 also possess certain coding exons (exon 2, 3 and exon 5-9) of the same lengths, which could also be found in cryl genes. Most importantly, zebrafish crylab and crylaa exhibited syntenic relationship with the human $C R Y 1$, while zebrafish cry2 showed syntenic relationship with the human CRY2 (Figure 3). Therefore, zebrafish has two cryl orthologous (crylaa and crylab) and two cryl homologous (crylba and crylbb) to human CRYI and one ortholog of human CRY2. On the contrary, zebrafish cry 4 and cry 5 were separated from any other cry genes in the phylogenetic analysis. They also have entirely different gene structures from any other cry genes. No syntenic relationship was found between zebrafish cry4 or cry5 and human $C R Y$ genes. Hence, cry 4 and cry 5 are paralogs of other zebrafish cry genes.

\section{RNA expression patterns}

RT-PCR was run to analyze RNA expression profiles of magr and cry genes. In adult zebrafish, transcripts of magr and all cry genes were examined in organs of the ectoderm (brain, eye and skin), mesoderm (heart, kidney and muscle), endoderm (liver) and female ovary. The expression of $m a g r$ was detected in all organs (Figure 4). In developing zebrafish embryos, magr RNA was detectable already at the 2-cell stage and until hatching (Figure 4), suggesting its 
maternal supply and continuous zygotic expression throughout embryogenesis. The transcripts of cry genes were highly abundant in the brain, eye and ovary but detectable also in the skin, heart and liver (Figure 4). In developing embryos, the transcripts of crylab, crylba, crylbb, cry2, cry 4 and cry5 were detected already at the 2-cell stage and also until hatching (Figure 4), suggesting their maternal inheritance and continuous zygotic expression throughout embryogenesis. In contrast, crylaa exhibited a different expression pattern. Its RNA was absent at the 2-cell stage embryos and became barely detectable at the morula stage, but it was easily detectable from gastrula stage onwards (Figure 4), implying that crylaa lacks maternal inheritance and commences zygotic expression from morula stage onwards. Taken together, a ubiquitous and high level RNA expression of magr accompanies differential expression patterns of cry genes in adult organs and developing embryos of zebrafish.

\section{Dispensability of magr for normal organogenesis}

To examine the role of magr in development, MOmagr, which is a morpholino designed to prevent the translation of magr mRNA, was microinjected into zebrafish embryos at the 1-cell stage. Survival rate and phenotype of the injected embryos were observed for up to $72 \mathrm{hpf}$. MOmagr-injected embryos exhibited a survival rate similar to that of control embryos (Table 1). MOmagr did not affect organogenesis, as MOmagr-injected embryos resembled control embryos in normal development, evidenced by scheduled development of major organs such as the eye, somites, heart and brain in MOmagr-injected embryos (Figure 5A-C'). Furthermore, we examined RNA expression of ectodermal marker genes (fgf8a and ot 2 ), mesodermal marker genes (gata4, gata5, has 2 and $n t l$ ) and endodermal marker gene (sall4) at 9, 24 and $48 \mathrm{hfp}$ and these genes displayed no obvious differential expression between MOmagr-injected embryos and control embryos (Figure 5D). In addition, the abundant expression of magr and cry genes in eye and brain indicates their potential role in the development of nerve system. We detected the expression level of three early neuronal markers sox2, elavl3, and gadlb (Schmidt et al., 2013), and found that the expression of those marker genes between MOmagr-injected and control embryos showed no obvious difference in 9, 30, and $48 \mathrm{hpf}$ (Figure S4 in Supporting Information). Therefore, magr appears to be dispensable for embryonic survival and early organogenesis of zebrafish.

\section{DISCUSSION}

The interest in magnet biology is twofold. One is to under-
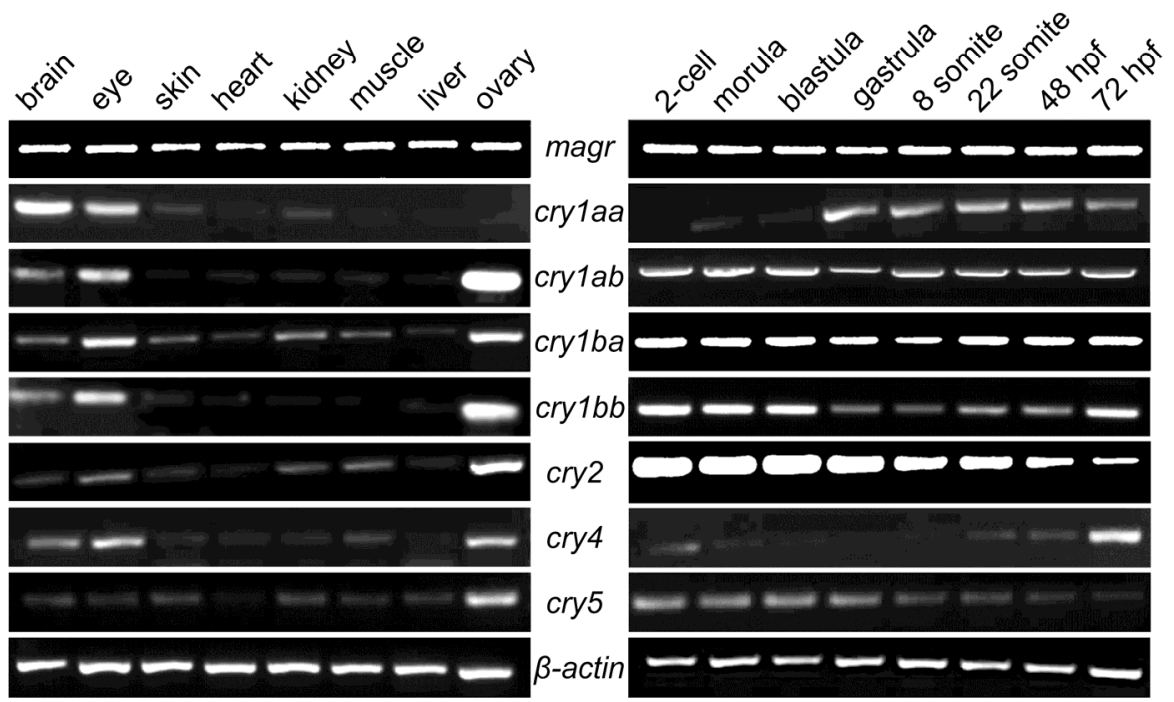

Figure 4 RNA expression of zebrafish magr and cry genes. left panel, Adult organs; right panel, Developing embryos. magr shows a high level of ubiquitous RNA expression in adult organs and developing embryos. All cry genes display RNA expression in the brain and eye as well as developing embryos.

Table 1 Development of MOmagr-injected zebrafish embryos ${ }^{\text {a) }}$

\begin{tabular}{|c|c|c|c|c|c|}
\hline \multirow{2}{*}{ Injection } & \multirow{2}{*}{ Embryos samples } & \multicolumn{4}{|c|}{ Embryos survived, $n(\%)^{*}$} \\
\hline & & $9 \mathrm{hpf}$ & $30 \mathrm{hpf}$ & Total fry & Normal fry $^{* * *}$ \\
\hline No & 90 & $86(96)$ & $84(93)$ & $84(93)$ & $82(98)$ \\
\hline
\end{tabular}

a) Embryos were noninjected or injected at the 1-cell stage with $2 \mathrm{ng}$ of MOmagr and observed at indicated stage. *,\% survival was obtained by comparisons between embryos sampled and embryos survived to stages of observation. **, \% normal fry was obtained by a comparison between numbers of normal fry and total fry at hatching. 

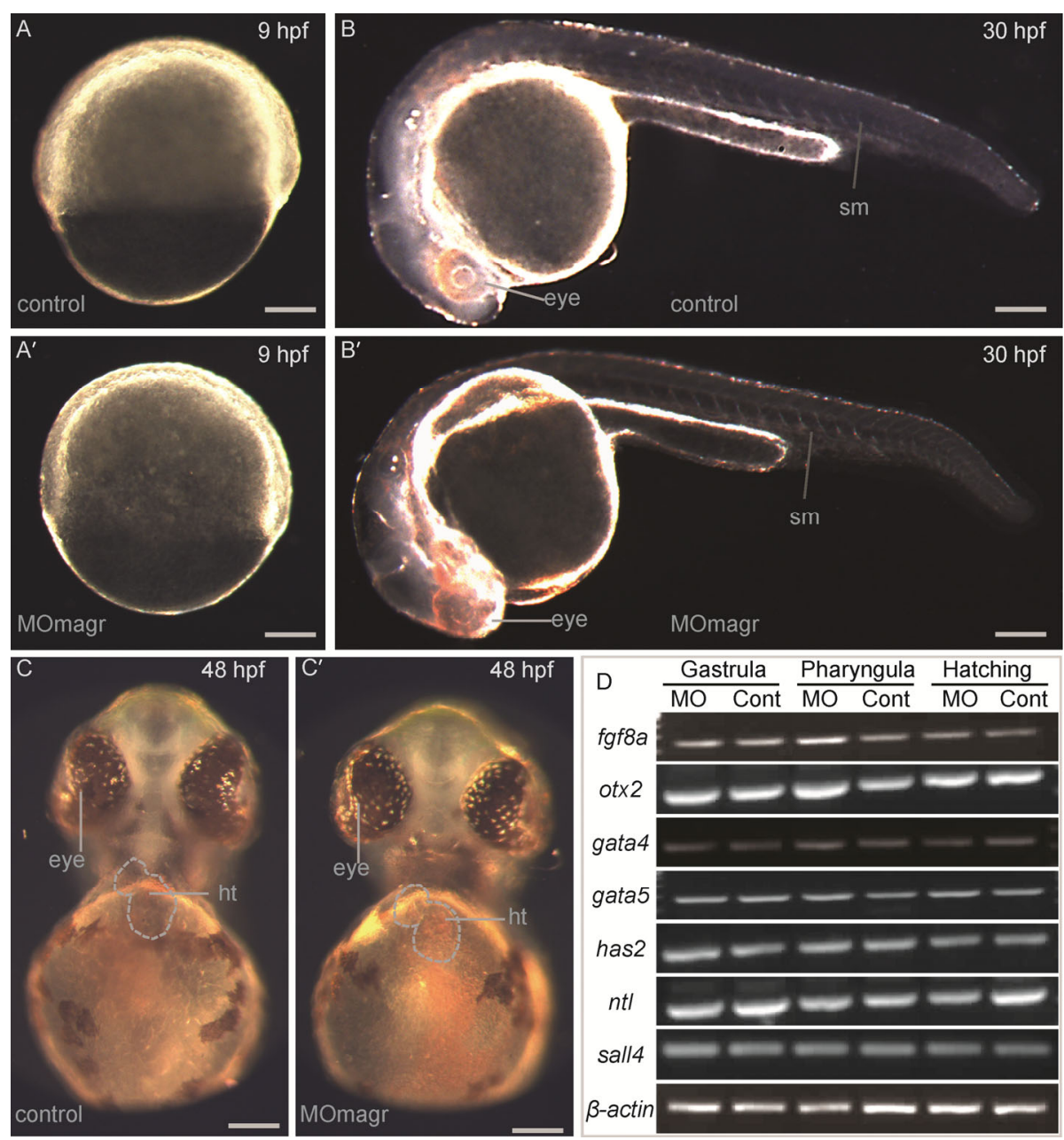

Figure 5 Normal development of magr-depleted embryos. Embryos were microinjected at the 1-cell stage with $2 \mathrm{ng}$ of MOmagr and observed at 9, 30 and 48 hpf. A-C, Control embryos without injection; A'-C', MOmagr-injected embryos; Showing normal organogenesis as evidenced by normal gastrulation (A and A') and organs such as eyes, somites and heart (sm, B and B'; ht, C and C'). Scale bars, $200 \mu \mathrm{m}$. D, RNA expression of three germ layer markers during embryogenesis. RNA expression of ectodermal marker genes ( fgf $8 a$ and otx2), mesodermal marker genes (gata4, gata5, has 2 and $n t l)$ and endodermal marker gene (sall4) dispalyed no obvious difference at gastrula $(9 \mathrm{hpf})$, pharyngula $(24 \mathrm{hpf})$, and hatching (48 hpf) stages between MOmagr-injected embryos (MO) and control embryos (Cont).

stand basic sciences via elucidating mechanisms underlying fundamental phenomena in nature such as animal migration. The other is to benefit human healthcare via elucidating mechanisms underlying the physiological role of magnetic fields towards the better practice of magnetomedicine. Zebrafish is an excellent model for developmental biology, functional genomics, and human diseases. In this study, we present data on the MagR-Cry system of zebrafish as a prelude to making use of this organism for magnet biology and physiology in vertebrates. We show that all the three human genes, namely MAGR, CRY1, and CRY2, have genuine orthologs in zebrafish.

We studied the expression pattern of magr and cry genes during developmental stages and in adult tissues of zebrafish. Base on previous studies, magnetic proteins are expected to express only in the eyes and brain. However, our RT-PCR result reveals that magr is expressed in all eight organs of zebrafish. We investigated human MAGR,
$C R Y 1$ and $C R Y 2$ expression in the expression atlas of EMBL and discovered that human MAGR, CRY1 and CRY2 also express in the same eight organs. Our explanation about the ubiquitous expression of magr and cry genes is that MagR-Cry complexes act as a magnetoreceptor in brain and eye, while MagR or Cry could have other biological functions in others organs, such as mitochondrial and cytosolic iron-sulfur cluster biogenesis (Muhlenhoff et al., 2011; Sheftel et al., 2012).

Using bioinformatics technique, we confirm that magr and cry2 are orthologs of human MAGR and CRY2; Crylaa and crylab are the orthologs of human CRYI; Crylba and crylbb are the homologs of human CRY1; the cry-like genes, cry 4 and cry5, are the paralogs of cry genes in zebrafish. In the representative species we have investigated, only one highly conservative magr gene exists in their genomes, implying that the magr gene keeps the original function. However, cry gene has been duplicated during the 
process of evolution, especially in zebrafish. The 7 cry genes found in zebrafish exhibit divergences in protein sequences, gene structures and expression patterns, implying that these genes may have different functions. In light of the previous study, there are two main functions of Cry proteins in animals. One function is to regulate circadian rhythms as components of the circadian pacemakers (Miyamoto and Sancar, 1998; Sancar, 2004; van der Horst et al., 1999; Zhu et al., 2008). The other is to act as a candidate lightdependent magnetoreceptor (Gegear et al., 2010; Maeda et al., 2012; Moller et al., 2004; Mouritsen et al., 2004). Interestingly, the Drosophila circadian clock hinges on the Cry-dependent magnetic sensitivity (Yoshii et al., 2009), suggesting that ancestry cry gene possesses both functions. In Zebrafish crylaa, crylab, crylba and crylbb, can function in circadian rhythms while cry2 and cry4 cannot (Kobayashi et al., 2000; Liu et al., 2015), suggesting that cry 2 and cry 4 might function as magnetoreceptors. It has been demonstrated that magnetosensitivity can be found in human and cockroaches Cry2 (Bazalova et al., 2016; Foley et al., 2011), and in pigeon and garden warbler Cry4 (Liedvogel and Mouritsen, 2010; Qin et al., 2016). We conducted protein sequence alignment between zebrafish Cry proteins with pigeon Cry4 and found that zebrafish Cry4 is maximally identical to pigeon Cry4 (70\%). Thus cry 2 and cry 4 could be good target genes in future investigation of zebrafish magnet biology.

Previous study reported that knocked down magr (iscal) results in profound anemia without affecting erythroid specification in zebrafish embryos (Nilsson et al., 2009), but no apparent effect on organogenesis and survival has been elucidated. In our gene knockdown experiments, MOmagr zebrafish did not display obvious abnormalities during early development when compared with control. The expression level of endoderm, mesoderm and ectoderm maker genes showed no evident difference between MOmagr and control embryos (Figure 5D). In addition, we found that the expression levels of its ligand cry genes also present no obvious variation (Figure S3 in Supporting Information). CRY is reported to be a deep brain circadian photoreceptor in fruit fly (Emery et al., 2000), and sponge eyes produce Cry proteins to communicate information of the light environment to the nervous system (Rivera et al., 2012), suggesting that Cry genes may play poteintial roles in neural system during embryogenesis. However, the expression level of early neuronal markers sox2, elavl3, and gad1b between MOmagr-injected and control embryos showed no significant difference. These results suggest that magr is dispensable for early organogenesis and that generating magr mutant to analyze its role in zebrafish is applicable. Such mutants could be used to study the physiological role of magnetic fields in zebrafish.

Magnetic fields have impacts on organismal life on the Earth and utility for treating diseases in magnetomedicine (Zhen and Xing-de, 2006). Transcranial magnetic stimula- tion based on the use of pulsed magnetic field, for example, is effective to treat disorders including multiple sclerosis, myelopathy, epilepsy, and stroke (Lefaucheur, 2005; Rossi et al., 2009). The molecular basis and mechanism of magnetomedicine remain to be elucidated. The availability of animal models will be invaluable to study the molecular basis and mechanism for magnet biology and magnet medicine. Our results that the MagR and Cry components are conserved from zebrafish to human demonstrate the suitability of zebrafish as a model organism for magnet biology and magnet medicine in vertebrates.

\section{MATERIALS AND METHODS}

\section{Fish}

Work with fish was carried out in strict accordance with the recommendations in the Guide for the Care and Use of Laboratory Animals of the National Advisory Committee for Laboratory Animal Research in Singapore and approved by this committee (Permit Number: 27/09). Zebrafish were maintained under an artificial photoperiod of 14-h/10-h light/darkness at $28^{\circ} \mathrm{C}$ and staged as described (Kimmel et al., 1995) .

\section{Phylogenetic, splice site and conserved syntenic analysis}

The magr and cry genes of zebrafish (Danio rerio), human (Homo sapiens), chicken (Gallus gallus), Chinese soft turtle (Pelodiscus sinensis), frog (Xenopus tropicalis) and fruitfly (Drosophila melanogaster) were retrieved from NCBI (http://www.ncbi.org/index.html) or Ensembl (http://www. ensembl.org/index.html). Exon boundaries of the coding regions within magr and cry genes were confirmed on NCBI. Multiple sequence alignments of the MagR and Cry proteins were generated using Clustal W (Thompson et al., 1994). The phylogenetic tree was constructed by Neighbor-Joining (NG) with Molecular Evolutionary Genetics Analysis version 6.0 (MEGA6.0) (Tamura et al., 2013). The phylogeny was tested by the bootstrap method with 500 replications. The substitution model was the JonesTaylor-Thornton (JTT) model. Numbers indicate bootstrap values. Using SyMap 4.0 (Soderlund et al., 2006) and biomart function in Ensembl, we determined the orthologs and co-orthologs of zebrafish to human genes in the chromosomal regions flanking $C R Y 1, C R Y 2$ and MAGR.

\section{RNA extraction and PCR}

Total RNA was extracted from zebrafish embryos and 3-month-old adult female zebrafish organs by using the TRIzol reagent from Invitrogen (USA) for cDNA synthesis as described (Fleige and Pfaffl, 2006). PCR was run in a 20 $\mu \mathrm{L}$ volume containing $10 \mathrm{ng}$ of cDNA reaction and primers listed in Table S1 in Supporting Information. PCR was run for 25 cycles ( $\beta$-actin) and 32 cycles for other genes of $10 \mathrm{~s}$ at $95^{\circ} \mathrm{C}, 30 \mathrm{~s}$ at $60^{\circ} \mathrm{C}$ and $30 \mathrm{~s}$ at $72^{\circ} \mathrm{C}$. The PCR products were analyzed on $1.0 \%$ agarose gels and documented on the 
Synoptics Bioimaging system (UK). All RT-PCR reactions were repeated three times.

\section{Morpholino oligo and microinjection}

MOmagr (AGAAGGCUUUAGGAAAGUUGAACAU), a morpholino oligo designed to block the translation of zebrafish magr mRNA, was synthesized by GeneTools (USA) and dissolved in water at $1 \mathrm{mmol} \mathrm{L}^{-1}$. Zebrafish embryos at the one-cell cell stage were injected with MOmagr at $2 \mathrm{ng}$ per embryos using a microinjector (Eppendorf, Femtojet). Injected embryos and control embryos without injection were reared in zebrafish egg water and regularly observed for survival and phenotypes.

Compliance and ethics The author(s) declare that they have no conflict of interest.

Acknowledgements This work was supported by the National Natural Science Foundation of China $(31572349,31272396)$ to Yuequn Wang, the China Scholarship Council (201406720012) to Xiyang Peng, the Cooperative Innovation Center of Engineering and New Products for Developmental Biology of Hunan Province (2013-448-6), and from the National Research Foundation of Singapore (NRF-CRP7-2010-03) to Yunhan Hong.

Bauer, S., and Klaassen, M. (2013). Mechanistic models of animal migration behaviour-their diversity, structure and use. J Anim Ecol 82, 498-508.

Bazalova, O., Kvicalova, M., Valkova, T., Slaby, P., Bartos, P., Netusil, R., Tomanova, K., Braeunig, P., Lee, H.J., Sauman, I., Damulewicz, M., Provaznik, J., Pokorny, R., Dolezel, D., and Vacha, M. (2016). Cryptochrome 2 mediates directional magnetoreception in cockroaches. Proc Natl Acad Sci USA 113, 1660-1665.

Cronin, T.W., Warrant, E.J., and Greiner, B. (2006). Celestial polarization patterns during twilight. Appl Opt 45, 5582-5589.

Dacke, M., Nilsson, D.E., Scholtz, C.H., Byrne, M., and Warrant, E.J. (2003). Animal behaviour: insect orientation to polarized moonlight. Nature 424, 33-33.

Egevang, C., Stenhouse, I.J., Phillips, R.A., Petersen, A., Fox, J.W., and Silk, J.R. (2010). Tracking of Arctic terns Sterna paradisaea reveals longest animal migration. Proc Natl Acad Sci USA 107, 2078-2081.

Emery, P., Stanewsky, R., Helfrich-Forster, C., Emery-Le, M., Hall, J.C., and Rosbash, M. (2000). Drosophila CRY is a deep brain circadian photoreceptor. Neuron 26, 493-504.

Fleige, S., and Pfaffl, M.W. (2006). RNA integrity and the effect on the real-time qRT-PCR performance. Mol Aspects Med 27, 126-139.

Foley, L.E., Gegear, R.J., and Reppert, S.M. (2011). Human cryptochrome exhibits light-dependent magnetosensitivity. Nat Commun 2, 356.

Gegear, R.J., Casselman, A., Waddell, S., and Reppert, S.M. (2008). Cryptochrome mediates light-dependent magnetosensitivity in Drosophila. Nature 454, 1014-1018.

Gegear, R.J., Foley, L.E., Casselman, A., and Reppert, S.M. (2010). Animal cryptochromes mediate magnetoreception by an unconventional photochemical mechanism. Nature 463, 804-807.

Heyers, D., Manns, M., Luksch, H., Güntürkün, O., and Mouritsen, H. (2007). A visual pathway links brain structures active during magnetic compass orientation in migratory birds. PLoS One 2, e937.

Holland, R.A., Thorup, K., Vonhof, M.J., Cochran, W.W., and Wikelski, M. (2006). Navigation: bat orientation using Earth's magnetic field. Nature 444, 702-702.

Kimchi, T., Etienne, A.S., and Terkel, J. (2004). A subterranean mammal uses the magnetic compass for path integration. Proc Natl Acad Sci USA 101, 1105-1109.

Kimmel, C.B., Ballard, W.W., Kimmel, S.R., Ullmann, B., and Schilling,
T.F. (1995). Stages of embryonic development of the zebrafish. Dev Dyn 203, 253-310.

Kobayashi, Y., Ishikawa, T., Hirayama, J., Daiyasu, H., Kanai, S., Toh, H., Fukuda, I., Tsujimura, T., Terada, N., Kamei, Y., Yuba, S., Iwai, S., and Todo, T. (2000). Molecular analysis of zebrafish photolyase/cryptochrome family: two types of cryptochromes present in zebrafish. Genes Cells 5, 725-738.

Lai, H. (1996). Spatial learning deficit in the rat after exposure to a $60 \mathrm{~Hz}$ magnetic field. Bioelectromagnetics 17, 494-496.

Lefaucheur, J. (2005). Transcranial magnetic stimulation: applications in neurology. Rev Neurol 161, 1121-1130.

Liedvogel, M., and Mouritsen, H. (2010). Cryptochromes-a potential magnetoreceptor: what do we know and what do we want to know? J R Soc Interface 7 Suppl 2, S147-S162.

Liu, C., Hu, J., Qu, C., Wang, L., Huang, G., Niu, P., Zhong, Z., Hong, F., Wang, G., Postlethwait, J.H., and Wang, H. (2015). Molecular evolution and functional divergence of zebrafish (Danio rerio) cryptochrome genes. Sci Rep 5, 8113.

Maeda, K., Robinson, A.J., Henbest, K.B., Hogben, H.J., Biskup, T., Ahmad, M., Schleicher, E., Weber, S., Timmel, C.R., and Hore, P.J. (2012). Magnetically sensitive light-induced reactions in cryptochrome are consistent with its proposed role as a magnetoreceptor. Proc Natl Acad Sci USA 109, 4774-4779.

Markov, M. (2015). XXIst century magnetotherapy. Electromagn Biol Med 34, 190-196.

Mather, J.G., and Baker, R.R. (1981). Magnetic sense of direction in woodmice for route-based navigation. Nature 291, 152-155.

Miyamoto, Y., and Sancar, A. (1998). Vitamin B2-based blue-light photoreceptors in the retinohypothalamic tract as the photoactive pigments for setting the circadian clock in mammals. Proc Natl Acad Sci USA 95, 6097-6102.

Moller, A., Sagasser, S., Wiltschko, W., and Schierwater, B. (2004). Retinal cryptochrome in a migratory passerine bird: a possible transducer for the avian magnetic compass. Naturwissenschaften 91, 585-588.

Mora, C.V., Davison, M., Wild, J.M., and Walker, M.M. (2004). Magnetoreception and its trigeminal mediation in the homing pigeon. Nature $432,508-511$.

Mouritsen, H., Janssen-Bienhold, U., Liedvogel, M., Feenders, G., Stalleicken, J., Dirks, P., and Weiler, R. (2004). Cryptochromes and neuronal-activity markers colocalize in the retina of migratory birds during magnetic orientation. Proc Natl Acad Sci USA 101, 14294-14299.

Muhlenhoff, U., Richter, N., Pines, O., Pierik, A.J., and Lill, R. (2011). Specialized function of yeast Isa1 and Isa2 proteins in the maturation of mitochondrial [4Fe-4S] proteins. J Biol Chem 286, 41205-41216.

Newton, I. (2010). The Migration Ecology of Birds (London, Academic Press).

Nilsson, R., Schultz, I.J., Pierce, E.L., Soltis, K.A., Naranuntarat, A., Ward, D.M., Baughman, J.M., Paradkar, P.N., Kingsley, P.D., and Culotta, V.C. (2009). Discovery of genes essential for heme biosynthesis through large-scale gene expression analysis. Cell Metabolism 10, 119-130.

Qin, S., Yin, H., Yang, C., Dou, Y., Liu, Z., Zhang, P., Yu, H., Huang, Y., Feng, J., Hao, J., Hao, J., Deng, L., Yan, X., Dong, X., Zhao, Z., Jiang, T., Wang, H.W., Luo, S.J., and Xie, C. (2016). A magnetic protein biocompass. Nat Mater 15, 217-226.

Rivera, A.S., Ozturk, N., Fahey, B., Plachetzki, D.C., Degnan, B.M., Sancar, A., and Oakley, T.H. (2012). Blue-light-receptive cryptochrome is expressed in a sponge eye lacking neurons and opsin. J Exper Biol $215,1278-1286$

Rossi, S., Hallett, M., Rossini, P.M., Pascual-Leone, A., and Safety of, T.M.S.C.G. (2009). Safety, ethical considerations, and application guidelines for the use of transcranial magnetic stimulation in clinical practice and research. Clin Neurophysiol 120, 2008-2039.

Sancar, A. (2004). Regulation of the mammalian circadian clock by cryptochrome. J Biol Chem 279, 34079-34082.

Schmidt, R., Strahle, U., and Scholpp, S. (2013). Neurogenesis in zebrafish-from embryo to adult. Neural Dev 8, 3.

Sheftel, A.D., Wilbrecht, C., Stehling, O., Niggemeyer, B., Elsasser, H.P., 
Muhlenhoff, U., and Lill, R. (2012). The human mitochondrial ISCA1, ISCA2, and IBA57 proteins are required for $[4 \mathrm{Fe}-4 \mathrm{~S}]$ protein maturation. Mol Biol Cell 23, 1157-1166.

Soderlund, C., Nelson, W., Shoemaker, A., and Paterson, A. (2006). SyMAP: a system for discovering and viewing syntenic regions of FPC maps. Genome Res 16, 1159-1168.

Tamura, K., Stecher, G., Peterson, D., Filipski, A., and Kumar, S. (2013). MEGA6: molecular evolutionary genetics analysis version 6.0. Mol Biol Evol 30, 2725-2729.

Thompson, J.D., Higgins, D.G., and Gibson, T.J. (1994). CLUSTAL W: improving the sensitivity of progressive multiple sequence alignment through sequence weighting, position-specific gap penalties and weight matrix choice. Nucleic Acids Res 22, 4673-4680.

van der Horst, G.T., Muijtjens, M., Kobayashi, K., Takano, R., Kanno, S., Takao, M., de Wit, J., Verkerk, A., Eker, A.P., van Leenen, D., Buijs, R., Bootsma, D., Hoeijmakers, J.H., and Yasui, A. (1999). Mammalian Cry1 and Cry2 are essential for maintenance of circadian rhythms. Nature 398, 627-630.

Vidal-Gadea, A., Ward, K., Beron, C., Ghorashian, N., Gokce, S., Russell, J., Truong, N., Parikh, A., Gadea, O., and Ben-Yakar, A. (2015). Magnetosensitive neurons mediate geomagnetic orientation in Caenorhab- ditis elegans. Elife 4, e07493.

Walleczek, J., and Liburdy, R.P. (1990). Nonthermal $60 \mathrm{~Hz}$ sinusoidal magnetic-field exposure enhances $45 \mathrm{Ca}^{2+}$ uptake in rat thymocytes: dependence on mitogen activation. FEBS Lett 271, 157-160.

Wang, G., and Wu, Z. (1997). Magnetochemistry and Magnetomedicine. (Beijing: The Publishing House of Ordnance Industry) pp. 82-90.

Wiltschko, R., and Wiltschko, W. (2006). Magnetoreception. Bioessays 28, $157-168$.

Yano, A., Ogura, M., Sato, A., Sakaki, Y., Shimizu, Y., Baba, N., and Nagasawa, K. (1997). Effect of modified magnetic field on the ocean migration of maturing chum salmon, Oncorhynchus keta. Mar Biol 129, 523-530.

Yoshii, T., Ahmad, M., and Helfrich-Forster, C. (2009). Cryptochrome mediates light-dependent magnetosensitivity of Drosophila's circadian clock. PLoS Biol 7, e1000086.

Zhen, Y., and Xing-de, W. (2006). New progress of research of biological effect of magnetic fields. Prog Modern Biomed 9, 032.

Zhu, H., Sauman, I., Yuan, Q., Casselman, A., Emery-Le, M., Emery, P., and Reppert, S.M. (2008). Cryptochromes define a novel circadian clock mechanism in monarch butterflies that may underlie sun compass navigation. PLoS Biol 6, e4.

Open Access This article is distributed under the terms of the Creative Commons Attribution License which permits any use, distribution, and reproduction in any medium, provided the original author(s) and source are credited.

\title{
SUPPORTING INFORMATION
}

\author{
Figure S1 Sequence alignment of MagR proteins. \\ Figure S2 Sequence alignment of Cry proteins. \\ Figure S3 RNA expression of cry genes during embryogenesis. \\ Figure S4 RNA expression of early neural maker genes.
}

Table S1 Zebrafish Genes and primers used for RT-PCR analyses

The supporting information is available online at life.scichina.com and link.springer.com. The supporting materials are published as submitted, without typesetting or editing. The responsibility for scientific accuracy and content remains entirely with the authors. 